\title{
Surface Enhanced Raman Spectroscopy Using Silver Nanoparticles: The Effects of Particle Size and Halide Ions on Aggregation
}

\author{
Garry P. Glaspell, ${ }^{1}$ Chen Zuo, ${ }^{2}$ and Paul W. Jagodzinski ${ }^{2,3}$
}

Received August 26, 2004

\begin{abstract}
A surface enhanced Raman spectroscopy (SERS) investigation of the aggregation of silver nanoparticles formed via LVCC with diameters in the range 5$50 \mathrm{~nm}$ were studied. It was found that with $647.1 \mathrm{~nm}$ excitation maximum enhancement is observed using particles with $11 \mathrm{~nm}$ diameters. Upon addition of sodium halides, enhancement is proportional to the polarizability of the anion. Maximum enhancement was observed when the concentration of the anion is approximately equal to the concentration of the adsorbate.
\end{abstract}

KEY WORDS: SERS; silver; nanoparticles; aggregation; halide.

\section{INTRODUCTION}

The surface enhanced Raman (SER) effect is currently explained using chemical (CM) and electromagnetic (EM) mechanisms. [1-4] Chemical effects provide enhancement via an increase in the molecular polarizability of the adsorbate due to the interaction of the adsorbate with the metal surface. Maximum chemical enhancement is observed when the substrate forms a monolayer on the metal of interest. Atomic scale structural features produce varying degrees of enhancement. Electromagnetic effects provide enhancement due to an increase in the electromagnetic field experienced by the molecule on the metal surface. The latter effect is paramount when investigating nanoparticles [5]. The size and shape of the particles, the specific metal, and hence the nature of the surface plasmons,

\footnotetext{
${ }^{1}$ Department of Chemistry, West Virginia University, Morgantown, WV 26506.

${ }^{2}$ Department of Chemistry \& Geochemistry, Colorado School of Mines, Golden, CO 80401.

${ }^{3}$ To whom correspondence should be addressed. E-mail: pwjag@mines.edu
} 
which can be defined as a sea of electrons with a particular resonance, contribute to the EM mechanism.

We have investigated the change in SER intensity of Rhodamine $6 \mathrm{G}$ (R6G) on silver nanoparticles as a function of initial particle size and as a function of aggregate formation with time. The latter work was performed both with and without the influence of halides ions. Faraday first reported that the addition of salts to metal sols resulted in aggregation of individual particles [6] and this approach has been applied to metal colloids in numerous SERS studies [7]. There are three mechanisms that describe the effect of adding salts to a metal colloid: activation, aggregation and desorption [8-10]. Activation is experienced at low concentrations of halides where the metal particles show an immediate increase in surface enhancement, but do not experience aggregation. Aggregation of the metal particles and the formation of "hot spots" is experienced at elevated halide concentrations. Desorption removes the adsorbate from the metal. A possible explanation provided by Hildebrandt [11] for the increased enhancement generated by activated and aggregated metal particles is a combination of the chemical and electromagnetic mechanisms.

Kniepp et al. [12-14], Brus et al. [15], and Nie and Emory [16, 17] have shown that not all particles are SER active and have reported that the aggregation of individual particles can lead to intense SER enhancement through the formation of "hot spots" (an intense plasmon resonance that exists between two particles in close proximity). Xa et al. [18] and Van Duyne et al. [19-21] have reported that the enhancement factors for a "hot spot" can be as high as $10^{13}$ to $10^{14}$ and that this could be responsible for single molecule detection.

Synthesis of various semiconductor and intermetallic nanoparticles via laser vaporization controlled condensation (LVCC) has been of particular interest [22]. This method utilizes well-defined conditions to produce nanoparticles of controlled size and composition without the need for chemical precursors or heat treatments. Specifically, a laser is used to produce a supersaturated metal vapor from a target inside a chamber. Convection currents, resulting from the top plate of the chamber being cooled and the bottom plate heated, affect the rate of nucleation of the metal vapor. The size of the particles can be controlled by the choice of laser power, chamber pressure, and temperature gradient [23-25]. Particles generated by this procedure are predominately spherical and have significantly less fluctuation in diameter (usually $\pm 3 \mathrm{~nm}$ ) compared to most reduction methods. Silver nanoparticles were generated using LVCC methodologies and SER data were collected using R6G since this adsorbate gives good enhancement on silver [26-28]. 


\section{EXPERIMENTAL}

Silver metal (99.98\%) was purchased from Alpha Aesar (Ward Hill, MA). Nanoparticles of silver were generated via LVCC in a home-built chamber that consists of two metal plates modified to provide uniform heating and cooling. To provide convection, the top plate was cooled with liquid nitrogen and the bottom plate was heated with circulating water. A Laser Photonics (Orlando, Fl) Nd/YAG laser was used to ablate the metal target. Altering the laser power, chamber pressure, and temperature gradient between the two plates results in the formation of particles of various sizes [29]. The size and shape of the particles were confirmed via transmission electron microscopy (TEM) using a JEOL 1220 (Tokyo, Japan) operating at $80 \mathrm{Kev}$. Figure 1 shows a representative particle size
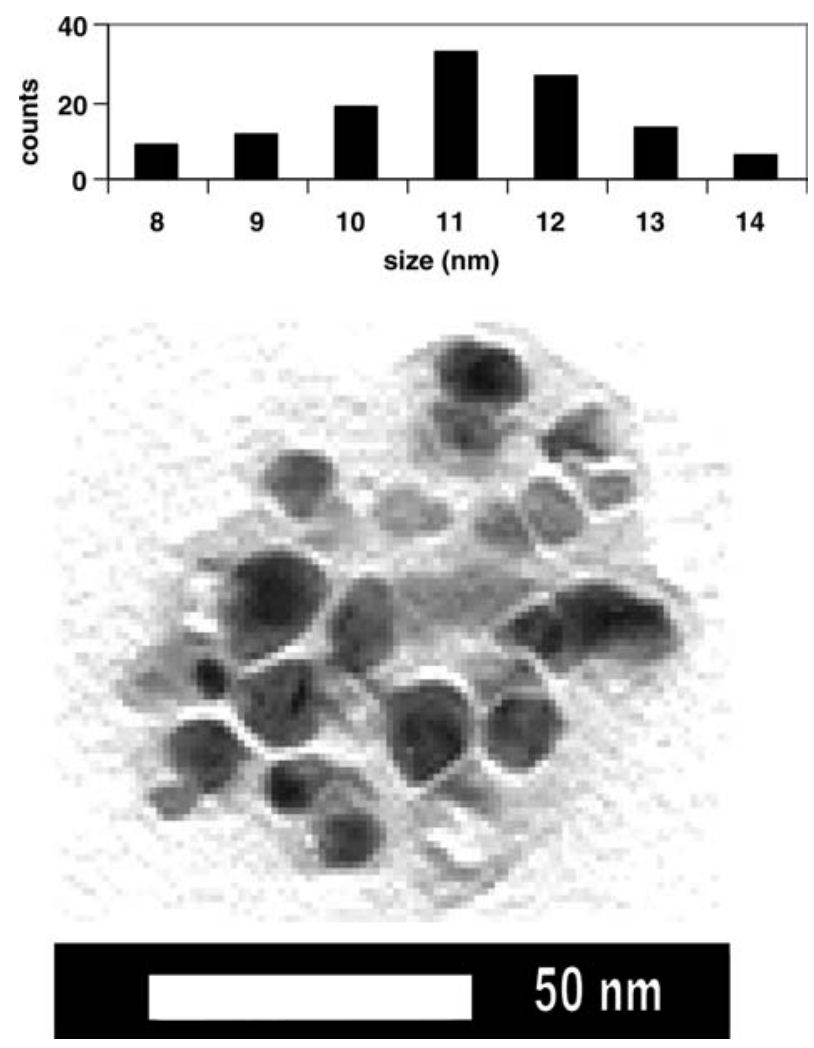

Fig. 1. Representative particle size histogram and TEM of $11 \mathrm{~nm} \mathrm{Ag} \mathrm{nanoparticles} \mathrm{pro-}$ duced via LVCC. 
histogram and TEM micrograph for the $11 \mathrm{~nm}$ sample. Immediately after formation, the silver particles $\left(\sim 5 \times 10^{-4} \mathrm{~g}\right.$ of particles $/ \mathrm{ml}$ of solution $)$ were placed in a test tube and mixed with a stock solution of R6G $\left(3.0 \times 10^{-4} \mathrm{M}\right)$ in ethanol. The R6G $(99 \%$; Lamda Physik, Fort Lauderdale, FL) and ethanol (99.9\%; Fisher Scientific, Pittsburgh, PA) were used without further purification.

Stock solutions of aqueous $\mathrm{NaF}, \mathrm{NaCl}, \mathrm{NaBr}$ and $\mathrm{NaI}$ were prepared at various concentrations and added to solutions of particles alone and particles with R6G. The concentrations of the anions in the solutions that were interrogated ranged from $1.7 \times 10^{-4} \mathrm{M}$ to $1.7 \times 10^{-1} \mathrm{M}$. The salts $(99 \%)$ were purchased from Fisher Scientific (Pittsburgh, PA) and used without further purification.

SER spectra were obtained using a SPEX Triplemate spectrometer (Edison, NJ) equipped with 1200 grooves $/ \mathrm{mm}$ gratings and an Oxford Instruments CCD detector cooled to $133 \mathrm{~K}$. A krypton ion laser (Lexel Laser, Fremont, CA) was used to provide excitation at $647.1 \mathrm{~nm}$. The laser power was set to $18 \mathrm{~mW}$ (at the sample) and the collection time was $5 \mathrm{~s}$. The intensities of the SER signals were normalized relative to the well-separated ethanol peaks at $2931 \mathrm{~cm}^{-1}$ (internal intensity standard). No signals due to ethanol were observed below $2900 \mathrm{~cm}^{-1}$. Spectra were collected up to $46 \mathrm{~h}$ after sample preparation after which oxidation of the silver surface obviated the possibility of obtaining reproducible data.

\section{RESULTS AND DISCUSSION}

Stock solution of R6G $\left(3.0 \times 10^{-4} \mathrm{M}\right)$ was added to the $\mathrm{Ag}$ particles until a maximum SER intensity was observed $\left(\sim 5 \times 10^{-4} \mathrm{~g} / \mathrm{mL}\right.$; due possibly to monolayer coverage since excess amounts of R6G decreased the SER intensity). Once the optimum intensity was reached SER spectra were collected at various times for each of the initial particle sizes. It is significant to note that at the concentration used the stock solution of R6G did not produce any observable peaks in the Raman spectrum. Over the range of particles tested $(5-50 \mathrm{~nm}$ in diameter) it was determined that $11 \mathrm{~nm}$ particles exhibited maximum spectral enhancement during a $46 \mathrm{~h}$ period (vide infra). Figure 2 shows the spectra for $11 \mathrm{~nm}$ particles as a function of time through $46 \mathrm{~h}$. Plots of normalized intensities versus time for 15,11 and $8 \mathrm{~nm}$ particles for four selected aromatic ring-based normal modes $\left(1650,1523,1365,1310 \mathrm{~cm}^{-1}\right)$ were generated. Figure 3 is an example of the plot generated from the data collected for the $1523 \mathrm{~cm}^{-1}$ stretching mode and shows a linear dependence of intensity with time. It is also significant to note that the increase in intensity as a function of time is not due to a lag in the R6G absorbing to the surface since the 


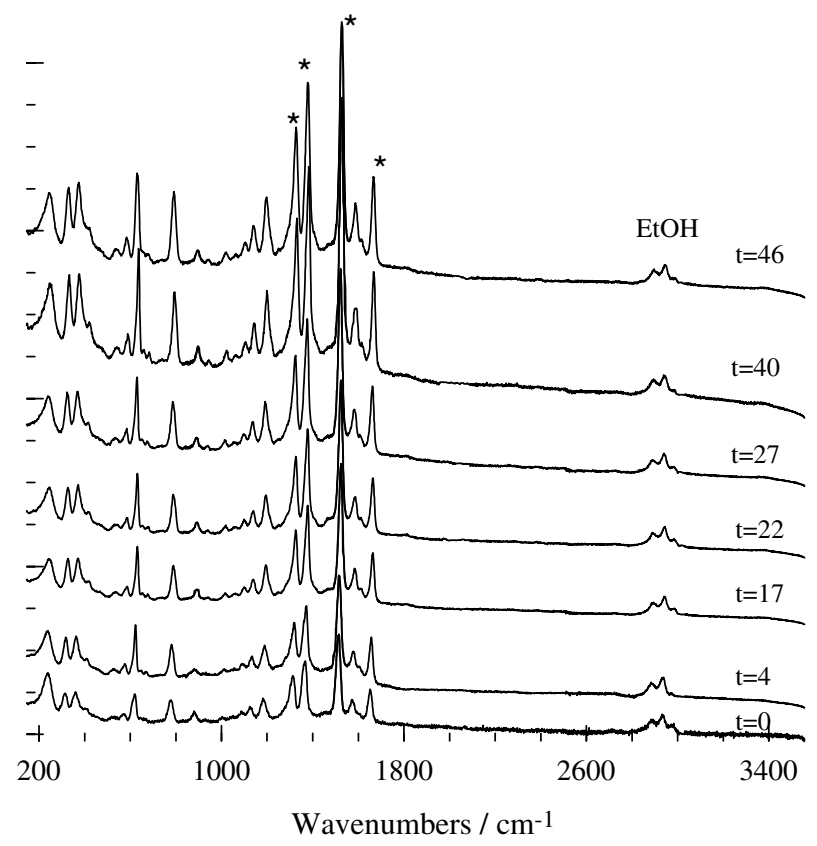

Fig. 2. Raman spectra (647.1 nm excitation) of $\mathrm{R} 6 \mathrm{G}\left(3 \times 10^{-4} \mathrm{M}\right)$ on $11 \mathrm{~nm} \mathrm{Ag}$ particles as a function of time (h). Ethanol peaks (EtOH) are only observed between 2900 and $3000 \mathrm{~cm}^{-1}$. Asterisks indicate which bands are being monitored.

stock solution of $\mathrm{R} 6 \mathrm{G}$ does not produce any observable peaks and enhancement is observed immediately after mixing the R6G with the silver particles. It was observed that the $11 \mathrm{~nm}$ particles exhibit the largest initial

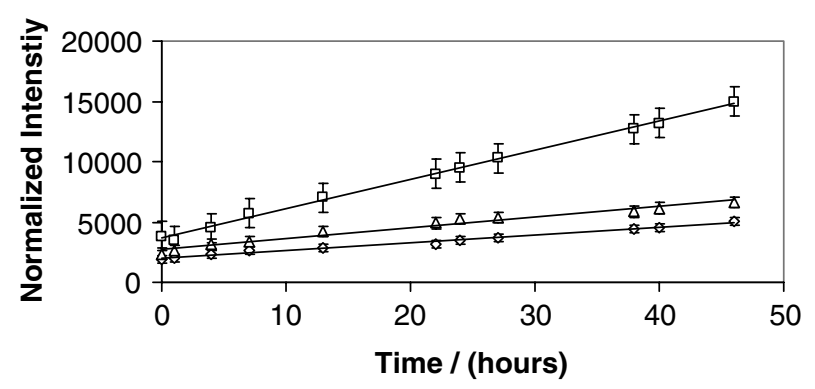

Fig. 3. Plot of normalized intensities of the R6G band at $1523 \mathrm{~cm}^{-1}$ as a function of time for $15 \mathrm{~nm}(\diamond), 11 \mathrm{~nm}(\square)$ and $8 \mathrm{~nm}(\triangle)$ diameter Ag particles. Error bars indicate the standard deviation of five trials. 
enhancement for each of the normal modes, although all three particle solutions do exhibit increased SER enhancement with time. Table I shows how the slopes differ among particle sizes for the four modes.

The observed enhancements could be explained via the chemical and electromagnetic effects of individual particles, hot spots of preformed clusters, or any combination of these. We can deduce that the chemical effect is minor since particles in the range of $40-50 \mathrm{~nm}$ do not exhibit a significant initial enhancement compared to the $11 \mathrm{~nm}$ particles, i.e., the initial spectra show a particle size dependent enhancement. We can also deduce that there are few preformed clusters since they would produce varying degrees of initial enhancement due to random numbers of "hot spots" which would be independent of particle size. We have observed reproducible initial enhancements and so preformed clusters are not a major contributor to the observed enhancements. Thus we can infer that the major contribution to the initial enhancement is the electromagnetic effect $[18,20]$.

In order to show that the intensity enhancements are related to aggregate formation we collected TEM images of the $11 \mathrm{~nm}$ particles throughout the period of SER spectra collection. These images are depicted in Fig. 4. Aggregation is also reflected in the red shift of the Raman signals with time $\left(15 \mathrm{~cm}^{-1}\right.$ shifts after $\left.46 \mathrm{~h}\right)$ [26]. As aggregates form, it is possible that "hot spots" are generated where the particles come in close contact [18]. We believe that this aggregation is caused by the R6G binding the particles together as reported by Kall and coworkers [30] for silver colloid particles. The enhancement is caused by a coupling of the single particle resonances and the localization of the potential drop due to the confined region between particles [31]. These authors show that with small interparticle separations there exists a localized region of ultrahigh electromagnetic enhancement which explains the "hot spots". Our

Table I. Slopes of the Intensity Versus Time Plots for Four Raman Lines of R6G as a Function of Size of the Silver Nanoparticles

\begin{tabular}{lccc}
\hline \multirow{2}{*}{ SER Line $\left(\mathrm{cm}^{-1}\right)$} & \multicolumn{3}{c}{ Particle Size $(/ \mathrm{nm})$} \\
\cline { 2 - 4 } & 15 & 11 & 8 \\
\hline 1650 & 23 & 198 & 48 \\
1523 & 63 & 243 & 88 \\
1365 & 38 & 238 & 77 \\
1310 & 37 & 182 & 77 \\
\hline
\end{tabular}




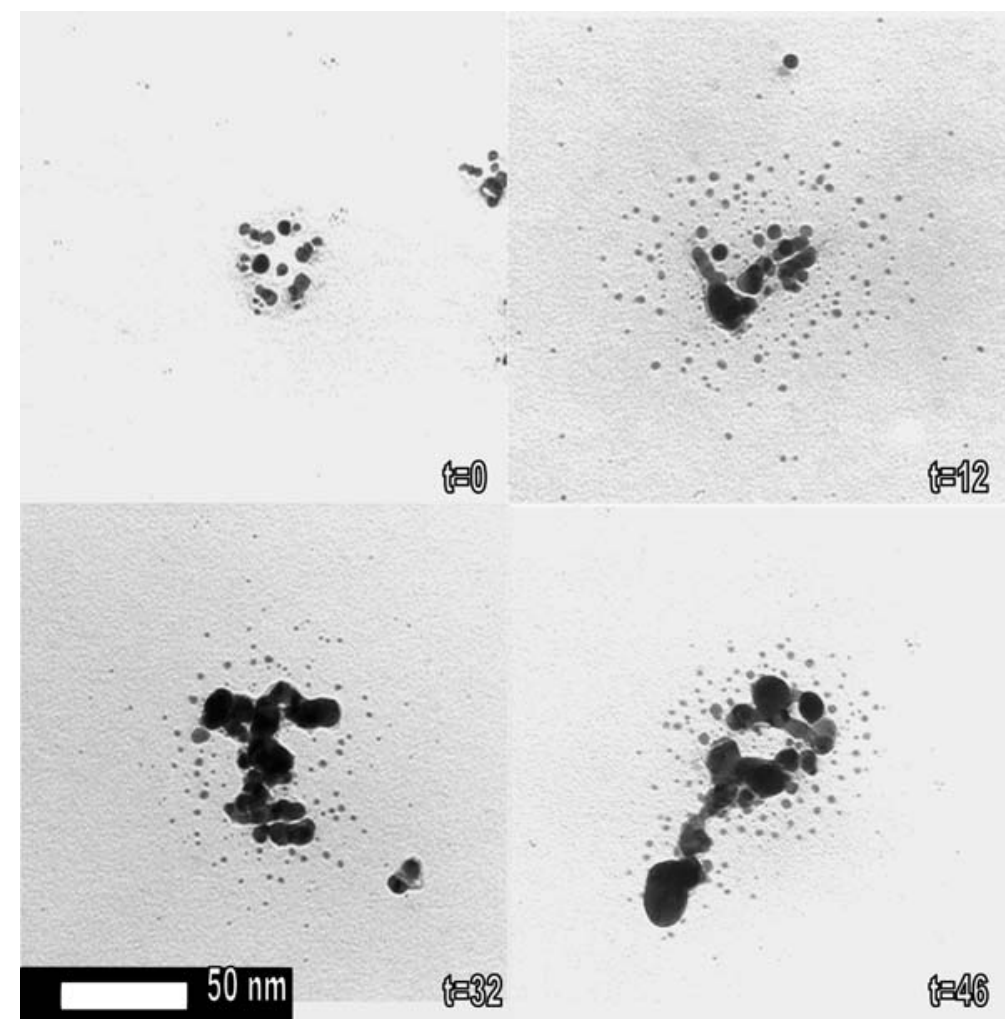

Fig. 4. TEM images of $11 \mathrm{~nm} \mathrm{Ag} \mathrm{particles} \mathrm{as} \mathrm{a} \mathrm{function} \mathrm{of} \mathrm{time} \mathrm{(h).} \mathrm{Formation} \mathrm{of} \mathrm{aggre-}$ gates is clearly visible.

data indicate that the energies of the "hot spots" associated with all three initial particle sizes increase their overlap with the excitation energy as the aggregates form since we observe increasing enhancement. Clearly the aggregation of $11 \mathrm{~nm}$ particles does this more efficiently when compared to the aggregation of $8 \mathrm{~nm}$ and $15 \mathrm{~nm}$ silver particles as evidenced by the intensity versus time slopes presented in Table I. After $46 \mathrm{~h}$ the relative intensities of the SER signals of the $11 \mathrm{~nm}$ particles are significantly stronger than their initial intensities.

Another observed trend is the difference in the slopes (see Table I) of the intensity versus time plots for the Raman bands arising from the four totally symmetric in-plane aromatic $\mathrm{C}-\mathrm{C}$ stretching normal modes [26]. The differences are attributed to the orientation of the $\mathrm{R} 6 \mathrm{G}$ with respect to the "hot spots" where stretching modes with a significant dipole 
moment vector perpendicular to the "hot spots" should exhibit the greatest enhancement [12-21]. One possible explanation is that with increasing numbers of aggregates the contributions from adjacent particles perturb the orientation of the adsorbed molecule into a more organized pattern between the particles.

We have also studied the effect of halide ions on the aggregation process. Figure 5 shows large intensity enhancements as a function of time for the four Raman bands after the addition of chloride ions (the final concentration of $\mathrm{R} 6 \mathrm{G}$ and $\mathrm{Cl}^{-}$are 0.003 and $0.0017 \mathrm{M}$, respectively) over a 7 h-period after which the particles become oxidized and intensity diminishes [23]. Table II shows the slopes of intensity versus time plots for the various salt additions to solutions containing R6G and $11 \mathrm{~nm}$ particles. The slopes are typically much more positive with halide ions present.

Again, one possible explanation of the enhancement as a function of time is the formation of "hot spots" due to aggregated particles. The significantly more positive slopes are attributed to the formation of R6Gadatom-anion surface complexes as proposed by Hildebrandt and Stockberger [26]. Comparing fluoride, chloride and bromide ions (omitting iodide because of the possibility of forming $\mathrm{I}_{3}^{-}$and other polyiodides due to trace amounts of water in the ethanol solution) enhancement increases proportionally with the polarizability of the anion. It is possible that at

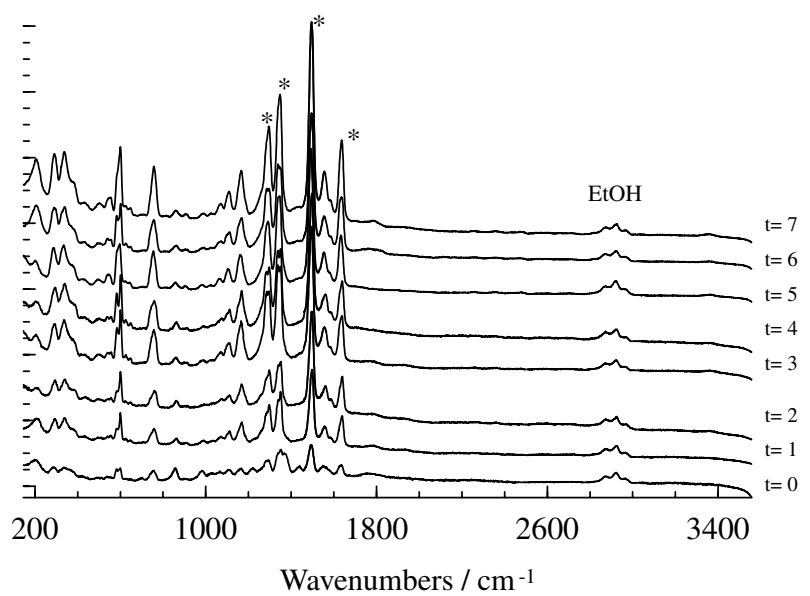

Fig. 5. Raman spectra (647.1 nm excitation) of R6G $\left(3.0 \times 10^{-4} \mathrm{M}\right)$ on $11 \mathrm{~nm} \mathrm{Ag} \mathrm{particles}$ with the addition of chloride ions $\left(1.7 \times 10^{-4} \mathrm{M}\right)$ as a function of time $(\mathrm{h})$. Ethanol peaks (EtOH) are only observed between 2900 and $3000 \mathrm{~cm}^{-1}$. Asterisks indicate which bands are being monitored. 
Table II. Slopes of the Intensity Versus Time Plots for Various Raman Lines for R6G $\left(3.0 \times 10^{-4} \mathrm{M}\right)$ Adsorbed on $11 \mathrm{~nm}$ Ag Particles for Various Halides

\begin{tabular}{|c|c|c|c|c|}
\hline \multirow[t]{2}{*}{ Salt Concentration (M) } & \multicolumn{4}{|c|}{ Raman Line } \\
\hline & $1310 \mathrm{~cm}^{-1}$ & $1365 \mathrm{~cm}^{-1}$ & $1523 \mathrm{~cm}^{-1}$ & $1650 \mathrm{~cm}^{-1}$ \\
\hline No salt & 181.8 & 238.3 & 242.7 & 197.9 \\
\hline \multicolumn{5}{|l|}{$\mathrm{F}^{-}$} \\
\hline .00017 & 556.5 & 607.8 & 782.6 & 554.3 \\
\hline .0017 & 522.7 & 658.5 & 740.1 & 574.2 \\
\hline .017 & 168.4 & 193.6 & 206.4 & 137.1 \\
\hline \multicolumn{5}{|l|}{$\mathrm{Cl}^{-}$} \\
\hline .00017 & 1764.5 & 2078.7 & 2947.9 & 1871.1 \\
\hline .0017 & 2300.4 & 2716.4 & 2967.3 & 2250.3 \\
\hline .017 & 663.3 & 880.6 & 914.6 & 587.4 \\
\hline .17 & 643.4 & 773.5 & 835.2 & 587.7 \\
\hline \multicolumn{5}{|l|}{$\mathrm{Br}^{-}$} \\
\hline .00017 & 2564.5 & 2729.3 & 3588.6 & 2704.2 \\
\hline .0017 & 2847.2 & 3268.3 & 4908.7 & 2411.8 \\
\hline .017 & 1315.8 & 1337.8 & 2751.9 & 1510.1 \\
\hline .17 & 1045.9 & 1227 & 1649.4 & 1111.8 \\
\hline \multicolumn{5}{|l|}{$\mathrm{I}^{-}$} \\
\hline .00017 & 1214.2 & 1450.4 & 1521 & 937.3 \\
\hline .0017 & 1718.5 & 1928.9 & 2295.7 & 1528.2 \\
\hline .017 & 490.3 & 553.5 & 595 & 462.2 \\
\hline .17 & 475.5 & 483.8 & 512.3 & 476.3 \\
\hline
\end{tabular}

The molarities reported are the final concentrations of the halide. The addition of $0.17 \mathrm{M} \mathrm{F}^{-}$ induced precipitation of R6G.

the "hot spot" oscillations of the plasmon resonance are able to permeate more effectively through the halide with the highest polarizability, thus extending the area providing the observed enhancement. To elucidate the effects of co-adsorption of the anions, the intensities of the Raman bands were observed immediately after the addition of each salt. The results are reported in Table III. Maximum enhancement is observed when the concentration of halide anions is approximately equal to the concentration of the adsorbate. This is different from previous results where a significant excess of anions were necessary for maximum enhancement using colloidal particle produced via a chemical reduction [32]. As shown in Table III, R6G Raman lines are observed immediately upon the addition of particles. This is contrast to results for typical particles prepared via chemical reduction processes in which the particles are allowed to age prior to 
Table III. Intensities of the Raman Peaks Measured, Relative to Ethanol, Immediately upon Addition of the Halide to a Solution of $3.0 \times 10^{-4}$ M R6G and $11 \mathrm{~nm}$ Silver Nanoparticles

\begin{tabular}{|c|c|c|c|c|}
\hline \multirow[t]{2}{*}{ Salt Concentration (M) } & \multicolumn{4}{|c|}{ Raman Line } \\
\hline & $1310 \mathrm{~cm}^{-1}$ & $1365 \mathrm{~cm}^{-1}$ & $1523 \mathrm{~cm}^{-1}$ & $1650 \mathrm{~cm}^{-1}$ \\
\hline No salt & 73.8 & 91.4 & 109.8 & 67.2 \\
\hline \multicolumn{5}{|l|}{$\mathrm{F}^{-}$} \\
\hline .00017 & 235.5 & 299.5 & 735.9 & 331.6 \\
\hline .0017 & 331.6 & 431.4 & 825.5 & 288.8 \\
\hline .017 & 241.5 & 305.6 & 529.2 & 182.5 \\
\hline \multicolumn{5}{|l|}{$\mathrm{Cl}^{-}$} \\
\hline .00017 & 1163.2 & 1574 & 1943.2 & 673.8 \\
\hline .0017 & 2095.5 & 2927.2 & 7766.2 & 1935.9 \\
\hline .017 & 1673.9 & 2371.8 & 4018.3 & 1717.7 \\
\hline .17 & 1170.1 & 1222.8 & 1441.3 & 981.3 \\
\hline \multicolumn{5}{|l|}{$\mathrm{Br}^{-}$} \\
\hline .00017 & 1329.2 & 1840.4 & 2464.5 & 786.8 \\
\hline .0017 & 5006.3 & 6532.5 & 11290.3 & 4065.8 \\
\hline .017 & 2862.7 & 4006.5 & 5752.9 & 2045.4 \\
\hline .17 & 1138.1 & 1605.3 & 2425.1 & 796.2 \\
\hline \multicolumn{5}{|l|}{$\mathrm{I}^{-}$} \\
\hline .00017 & 216.6 & 342.9 & 602.3 & 156.1 \\
\hline .0017 & 670.9 & 931.5 & 1668.5 & 438.7 \\
\hline .017 & 267.7 & 381.8 & 616.9 & 157.4 \\
\hline .17 & 118.6 & 180.4 & 429.5 & 183 \\
\hline
\end{tabular}

observation of strong SER signals. In addition, the repeatability of the initial enhancement is attributed to the uniform size and shape of the particles produced via LVCC and not generally observed with particles produced via chemical reduction. This is most probably due to the absence of variable surface features that lead to variations in the observed SERS for the chemical reduction particles. Finally, the surfaces on the particles are "clean" and have no adsorbed species from the reduction process. The decrease in intensity when the halide concentration is high is assumed to be due to coverage of the SERS active regions by anions rather than R6G as described by the chemical and electromagnetic mechanisms. Considering the trend in the slopes for the intensity versus time plots for the four aromatic modes (shown in Table II), we find remarkable consistency for each anion at each concentration relative to particles without the addition of halides. Namely $\mathrm{m}_{1523}>\mathrm{m}_{1365}>\mathrm{m}_{1650}>\mathrm{m}_{1310}$. This leads to the assumption that the addition of halides to the $\mathrm{R} 6 \mathrm{G} /$ silver nanoparticle 
solution does not change the orientation of the R6G. One indication of aggregation is the red shift of the Raman signals as a function of time [21]. In the absence of halide ions the peaks shifted approximately $15 \mathrm{~cm}^{-1}$ after $46 \mathrm{~h}$, whereas, the same red shift of $15 \mathrm{~cm}^{-1}$ was observed after $26 \mathrm{~h}$ with the addition of bromide ions $\left(1.7 \times 10^{-3} \mathrm{M}\right)$.

\section{CONCLUSION}

We have observed that the range of diameters produced for Ag particles needed for significant SERS enhancement is relatively narrow $(11 \pm$ $3 \mathrm{~nm}$ ) for $647.1 \mathrm{~nm}$ excitation for particles ranging in size from 5 to $50 \mathrm{~nm}$. The main contribution to enhancement is the electromagnetic mechanism since particles outside the range exhibit little or no enhancement. Since the excitation wavelength was invariant, we attribute the increased SER enhancement with time to be due to particle aggregation which is supported by TEM images as a function of time. The interaction of nanoparticle aggregates could provide an increase in the number of hot spots. Thus the EM mechanism must contribute significantly to the intensity although more rigorous studies of the surface morphology need to be conducted to confirm the dependence of this effect. Future work will involve the determination of the optimum size of Ag particles for SER enhancement at various excitation wavelengths.

We have also observed that the effect of halide ion concentration on SER enhancement is related to the concentration of adsorbate. Maximum enhancement is observed when the concentration of the halide is approximately equal to that of the adsorbate when monitoring the effects of aggregation and co-adsorption on the SER signal. In comparing the enhancement provided by various halides (fluoride, chloride and bromide) we have also observed a dependence on the polarizability of the anion involved. Furthermore, the invariance of the slopes of the intensity versus time plots with and without the presence of halide ions leads to the assumption that the addition of halides does not alter the orientation of R6G on the surface.

In summary, we have shown that maximum SER enhancement for R6G adsorbed on silver nanoparticles produced via LVCC is obtained with $11 \mathrm{~nm}$ particles when using $647 \mathrm{~nm}$ excitation. We have observed an increase in enhancement as a function of time due to aggregation caused by the adsorbate and an increase in enhancement as a function of time due to the formation of R6G-adatom-anion surface complexes. We believe that the differences between our results and previously reported results are due to the differences in particles produced via LVCC and colloidal particles produced via chemical reduction methods. 


\section{ACKNOWLEDGMENTS}

The authors express their gratitude to Dr. George Schatz (Northwestern University), Dr. Michael Kall (Chalmers University of Technology), Dr. Fred King (West Virginia University), Dr. Matthew Scanlon (Fairmont State University) and Michael Jacobs (Colorado School of Mines) for valuable consultations and helpful discussions.

\section{REFERENCES}

1. C. V. Raman and K. S. Krishnan (1928). Nature 121, 501.

2. A. Skoog, F. J. Holler and T. A. Nieman, Principles of Instrumental Analysis, 5th edn. (Saunders College Publishing, Philadelphia, 1998).

3. M. Fleischmann, P. J. Hendra and A. McQuillan (1974). J. Chem. Phys. Lett. 26, 163.

4. R. K. Chang and T. E. Furtak Surface Enhanced Raman Scattering (Plenum Press, New York 1982).

5. M. Kerker, D. Wang and S. Chew (1980). Appl. Opt. 19, 3373.

6. M. Faraday (1857). Trans. R. Soc. London 147, 145.

7. X. Mie (1908). Ann. Physik. 26, 377.

8. H. Wetzel and H. Gerischer (1980). Chem Phys. Lett. 76, 460.

9. E. J. Liang, C. Engert and W. Kiefer (1995). Vib. Spectrosc. 8, 435.

10. M. Kerker, O. Siiman and D. Wang (1984). J. Phys. Chem. 15 (88), 3168.

11. P. Hildebrandt, S. Kellar, A. Hoffmann, F. Vanhecke and B. Schrader (1993). J. Raman Spectrosc. 24, 791.

12. K. Kneipp, H. Kneipp, I. Itzkan, R. R. Dasari and M. S. Feld (1999). Chem. Phys. 247 (1), 155 .

13. K. Kneipp, H. Kneipp, R. Manoharan, I. Itzkan, R. R. Dasari and M. S. Feld (1998). J. Raman Spectrosc. 29 (8), 743.

14. K. Kneipp, H. Kneipp, G. Deinum, I. Itzkan, R. R. Dasari and M. S. Feld (1998). Appl. Spectrosc. 52 (2), 175.

15. A. M. Michaels, J. Jiang and L. Brus (2000). J. Phys. Chem. B 104 (50), 11965.

16. S. R. Emory and S. Nie (1997). Anal. Chem. 69 (14), 2631.

17. S. Nie and S. R. Emory (1997). Science 275 (5303), 1102.

18. H. Xa, J. Aizpurua, M. Kall and P. Apell (2000). Phys. Rev. E:Stat. Phys., Plasmas, Fluids, Relat. Interdiscip. Top. 62 (3-B), 4318.

19. J. C. Hulteen, D. A. Treichel, M. T. Smith, M. L. Duval, T. R. Jensen and R. P. Van Duyne (1999). J. Phys. Chem. B 103 (19), 3854.

20. T. R. Jensen, M. L. Duval, K. L. Kelly, A. A. Lazarides, G. C. Schatz and R. P. Van Duyne (1999). J. Phys. Chem. B 103 (45), 9846.

21. M. D. Malinsky, K. L. Kelly, G. C. Schatz and R. P. Van Duyne (2001). J. Phys. Chem. B 105 (12), 2343.

22. S. S. Li, S. J. Silvers and M. S. El-Shall (1997). J. Phys. Chem. B 101, 1794.

23. W. Z. Wang, I. N. Germanenko, G. Glaspell and M. S. El-Shall (2000). Clusters Nanostruct. Interfaces, Proc. Int. Symp. 83.

24. M. S. El-Shall, NASA Conf. Publ., (NASA Microgravity Materials Science Conference, 1998) p. 181.

25. M. S. El-Shall, S. Li, D. Graiver and U. Pernisz (1996). ACS Symp. Ser. 622 (Nanotechnology), 79 . 
26. P. Hildebrandt and M. Stockburger (1984). J. Phys. Chem. 88 (24), 5935.

27. K. Kneipp, Y. Wang, R. R. Dasari and M. S. Feld (1995). Appl. Spectrosc. 49 (6), 780.

28. S. R. Emory, W. E. Haskins and S. Nie (1998). J. Am. Chem. Soc. 120 (31), 8009.

29. M. S. El-Shall, W. Slack, W. Vann, D. Kane and D. Hanley (1994). J. Phys. Chem. 98 (12), 3067.

30. H. Xu, E. J. Bjerneld, M. Kall and L. Borjesson (1999). Phys. Rev. Lett. 83, 4357.

31. H. X. Xu, J. Aizpurua, M. Kall and P. Apell (2000). Phys. Rev. E 6, 4318.

32. K. Kneipp, R. Roth, C. Engert and W. Kiefer (1993). Chem. Phys. Lett. 207, 450. 